\title{
Rings sliding on a honeycomb network: Adsorption contours, interactions, and assembly of benzene on $\mathrm{Cu}(111)$
}

\author{
K. Berland, ${ }^{1, *}$ T. L. Einstein, ${ }^{2, \dagger}$ and P. Hyldgaard ${ }^{1, \dagger}$ \\ ${ }^{1}$ Department of Microtechnology and Nanoscience, MC2, Chalmers University of Technology, SE-41296 Göteborg, Sweden \\ ${ }^{2}$ Department of Physics, University of Maryland, College Park, Maryland 20742-4111, USA
}

(Received 20 July 2009; revised manuscript received 10 September 2009; published 14 October 2009)

\begin{abstract}
Using a van der Waals density functional (vdW-DF) [Phys. Rev. Lett. 92, 246401 (2004)], we perform $a b$ initio calculations for the adsorption energy of benzene $(\mathrm{Bz})$ on $\mathrm{Cu}(111)$ as a function of lateral position and height. We find that the vdW-DF inclusion of nonlocal correlations (responsible for dispersive interactions) changes the relative stability of eight binding-position options and increases the binding energy by over an order of magnitude, achieving good agreement with experiment. The admolecules can move almost freely along a honeycomb web of "corridors" passing between fcc and hcp hollow sites via bridge sites. Our diffusion barriers (for dilute and two condensed adsorbate phases) are consistent with experimental observations. Further vdW-DF calculations suggest that the more compact (hexagonal) Bz-overlayer phase, with lattice constant $a$ $=6.74 \AA$, is due to direct $\mathrm{Bz}-\mathrm{Bz}$ vdW attraction, which extends to $\sim 8 \AA$. We attribute the second, sparser hexagonal Bz phase, with $a=10.24 \AA$, to indirect electronic interactions mediated by the metallic surface state on $\mathrm{Cu}(111)$. To support this claim, we use a formal Harris-functional approach to evaluate nonperturbationally the asymptotic form of this indirect interaction. Thus, we can account well for benzene self-organization on $\mathrm{Cu}(111)$.
\end{abstract}

DOI: 10.1103/PhysRevB.80.155431

PACS number(s): 68.43.-h, 73.20.-r, 71.15.Mb, 73.90.+f

\section{INTRODUCTION}

With adsorption of atoms and of simple molecules on metals relatively well understood, interest has turned to the adsorption of organic molecules. ${ }^{1}$ There are many technological motivations for studying adsorption of electronically functional organic overlayers, e.g., organic light-emitting diodes offer great promise for displays. ${ }^{2}$ Acenes and acene derivatives are of particular interest, not just because of their importance in organic semiconductors but also because of the self-organized structures that they form on surfaces.

Arguably the most dramatic example of the latter is the giant regular honeycomb network formed by anthraquinone (AQ) on $\mathrm{Cu}(111){ }^{3}$ The pore diameter is unprecedentally large, over $5 \mathrm{~nm}$, and each cell encloses over 200 uncovered $\mathrm{Cu}$ surface atoms. Our understanding is that the adsorption process is primarily due to van der Waals (vdW) interactions, the short-range arrangement is due to hydrogen bonding from an aromatic hydrogen on one AQ to an oxygen of a carbonyl group on its neighbor; we believe that the longrange regularity can be attributed to indirect interactions mediated by the metallic (Shockley) surface state centered around the zone center of noble-metal (111) surfaces, ${ }^{4}$ in a mechanism analogous to that documented for atomic adsorbates. $^{5-10}$

In a first attempt to understand the details of the adsorption and self-organization of acene derivatives, we here consider the simplest member of this family, the monocyclic aromatic ring benzene $(\mathrm{Bz})$. Adsorption of $\mathrm{Bz}$ on $\mathrm{Cu}(111)$ displays remarkable molecular-adsorption dynamics with observations $^{11-17}$ of high mobility of individual Bz molecules at low temperatures, as well as a possibility for the $\mathrm{Bz}$ adsorbates to condense into two coexisting adsorbate-overlayer phases, $\mathrm{C} 1$ and $\mathrm{C} 2$, characterized by hexagonal nets with lattice constants $10.24 \AA$ and $6.74 \AA$, respectively. For Bz, as for AQ, the adsorption energy arises primarily from $\mathrm{vdW}$ interactions with conduction electrons on the $\mathrm{Cu}$ surface, with very small orbital changes and substrate-adsorbate charge transfers. ${ }^{18}$

The case of $\mathrm{Bz}$ on $\mathrm{Cu}(111)$, Fig. 1, provides a forum at which to show the power of a recently developed vdW density functional (vdW-DF) (Refs. 19-21) to describe molecular physisorption on a system with extremely low corrugation. Systems with low corrugation are interesting and challenging since they leave the adsorbates free to respond to weak direct and indirect mutual interactions which must therefore be described and analyzed in detail. Traditional density-functional theory (DFT) calculations, based on local or semilocal approximations for the functional, provide detailed information about the variations in atomic-adsorbate energies ${ }^{22-24}$ but cannot account for dispersive interactions. ${ }^{21,25,26}$ Thus, traditional DFT calculations have only a limited ability to compare binding energies, diffusion and rotation barriers for the molecular adsorption and does not permit an analysis of the adsorbate self-organization. In particular, the generalized gradient approximation (GGA) has proved inadequate for $\mathrm{Bz}$ on $\mathrm{Cu}(111) .{ }^{27}$ In this paper we report vdW-DF calculations of the subtle variation in the adsorption energy of individual molecules with bonding site, of the intermolecular vdW binding, and of the energy barriers for adsorbate dynamics at different adsorbate condensations.

Furthermore, we use the vdW-DF results to analyze and possibly interpret the self-organization mechanisms observed for $\mathrm{Bz}$ adsorption on $\mathrm{Cu}(111) .{ }^{15}$ Assuming that the admolecules form a close-packed (hexagonal) structure, we determine its lattice constant from the minimum of the interaction potential. For the more compact $C 2$ phase, our vdW-DF calculations of the direct $\mathrm{vdW}$ binding indicate a preference for a lattice constant of $6.9 \AA$. Given the tendency of the 


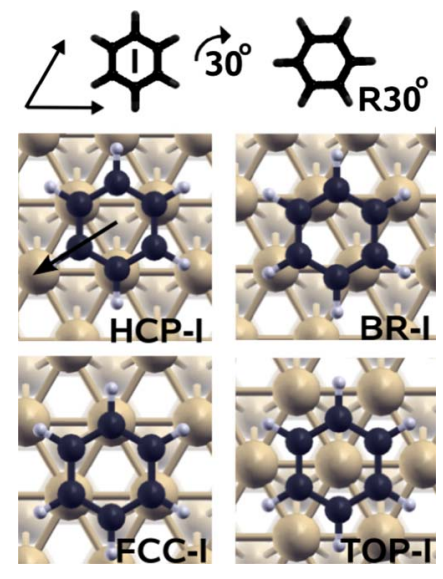

FIG. 1. (Color online) Schematics of the eight high-symmetry configurations of adsorbed $\mathrm{Bz}$ on $\mathrm{Cu}(111)$. High-symmetry configurations are found when the center of the molecules align with the traditional characteristic atomic-adsorption sites of the $\mathrm{Cu}(111)$ surface (four main panels). In the illustrated $I$ ("in-phase") orientation, one of the two equilateral triangles formed by alternate C's of the ring sits above three $\mathrm{Cu}$ atoms in the surface for hcp and fcc; for BR and TOP, these three carbons can be similarly aligned via a translation. Orientation $R 30^{\circ}$ differs from orientation $I$ by a $30^{\circ}$ rotation of the $\mathrm{Bz}$ adsorbate, as shown in the top pair of sketches. The arrow in upper left main panel illustrates a possible diffusion path that connects the high-symmetry sites, running from an $\mathrm{HCP}-I$ site, over a bridge site BR- $I$, then over an FCC- $I$ site, and finally to a TOP- $I$ site. A similar path could occur for the $R 30^{\circ}$ orientation.

vdW-DF method to slightly overestimate binding separations, ${ }^{21,28}$ this result suggests that the $\mathrm{C} 2$ phase can be interpreted as a direct, vdW-bound molecular-lattice structure. Explicit vdW-DF predictions of the preferred Bz orientations and of rotational barriers may offer experimental tests of this interpretation. For the less dense Cl phase we find that the lattice spacing lies beyond the range of substantial bonding via the direct intermolecular vdW binding. However, use a Harris-functional scheme ${ }^{8,29}$ formally extends our DFT analysis and shows that the $\mathrm{C} 1$ lattice spacing agrees excellently with the first energy minimum of the indirect electronic interaction ${ }^{6-8}$ mediated by the $\mathrm{Cu}(111)$ metallic surface-state (Shockley) band. Since we have explicitly verified that the $\mathrm{Cu}(111)$ surface state survives the addition of $\mathrm{Bz}$ molecules, we find it plausible that this indirect electronic interaction underpins the self-organization in the $\mathrm{C} 1$ phase.

This paper has the following plan: in Sec. II we summarize the vdW-DF theory and formal Harris-functional evaluation that we use along with various computational details. In Sec. III we present our results for adsorption energies, diffusion barriers, and barriers to rotation. In the next section we discuss these results, emphasizing possibilities for crystal organization. Section V contains our conclusions.

\section{DENSITY-FUNCTIONAL THEORY}

A recent vdW density functional, vdW-DF, ${ }^{19,20}$ has proved useful in describing a variety of sparse matter, ${ }^{21}$ i.e., systems which contain important regions of low electron density where dispersive interactions contribute significantly to the materials' coherence. The vdW-DF method provides a transferable account of general materials and bindings, ranging, e.g., from the all-covalent $\mathrm{C}-\mathrm{C}$ bonding within a graphene sheet to the interlayer dispersive bonding in graphite and graphite intercalates. ${ }^{30}$ DFT calculations using the vdW-DF has successfully characterized the adsorption of $\mathrm{Bz}$ on the semimetal graphene ${ }^{31}$ and on the semiconductors silicon ${ }^{32}$ and $\mathrm{MoS}_{2}{ }^{21} \mathrm{~A}$ vdW-DF study also documents that vdW interactions (nonlocal correlations) significantly affect the anchoring of phenol on an oxide. ${ }^{33}$ There are fewer applications for the interaction between organic molecules and metal surfaces but very recent examples include the adsorption on $\mathrm{Cu}(110)$ of triophene by Sony et al. ${ }^{34}$ and of $\mathrm{Bz}$ and other organic molecules by Atodiresei et al. ${ }^{35}$

We describe the physisorption and two-dimensional assembly of $\mathrm{Bz}$ on $\mathrm{Cu}(111)$ within $\mathrm{DFT}$ by combining largescale vdW-DF calculations and a formal Harris-functional evaluation, ${ }^{8}$ for: (a) adsorption of isolated $\mathrm{Bz}$ molecules and (b) direct and indirect (substrate-mediated) interactions between Bz adsorbates. Brute-force vdW-DF calculations treating the physisorption and the interadsorbate interactions in one giant unit cell are not computationally tractable for $\mathrm{Bz}$ adsorbates on $\mathrm{Cu}(111)$. Instead, we apply large-scale vdW-DF calculations to characterize the molecular adsorption both in the case of a frozen surface and in the presence of adsorption-induced surface relaxations. A separate set of vdW-DF calculations investigate the direct molecular interaction between pairs and in a regular three-dimensional lattice by ignoring the coupling to the substrate and by focusing strictly on the two-dimensional arrangement of adsorbates. Finally, we use a general, analytical Harris-functional evaluation $^{8,29}$ for a nonperturbative treatment of the indirect electronic interaction between adsorbates to formally complete an approximative DFT characterization of the adsorbate interaction and of the two-dimensional self-organization of $\mathrm{Bz}$ on $\mathrm{Cu}(111)$. This indirect electronic interaction is oscillatory and very long range; it arises from interference of Friedel oscillations produced by the strong adsorbate-induced scattering of surface-state electrons. ${ }^{5,8,36}$ For atomic adsorbates, the indirect electronic interaction can be described in traditional DFT implementations using very-large-scale unit cells $;{ }^{10}$ the present vdW-DF study must, in practice, rely on an implicit parameterization given by the detailed nature of the adsorbate-induced scattering. , $8,37^{-3}$

Our splitting of the physisorption and assembly problems permits us to provide explicit vdW-DF determinations for some properties but can, naturally, only serve as an approximate characterization of other properties. Our description of the combined interactions of the adsorbates ignores, for example, the contributions to the direct vdW binding between molecules from small, adsorption-induced changes in the electron-density distribution. Such effects should be present even if our use of a Bader analysis ${ }^{38,39}$ documents that the total adsorbate-to-substrate charge transfer vanishes. Also, the precise nature of the surface-state scattering induced by $\mathrm{Bz}$ adsorption is not known. Lacking a detailed experimental characterization, we can only apply our formal Harrisfunctional evaluation to provide a qualitative description of the long-range indirect electronic interaction ${ }^{5-8}$ between adsorbates. Nevertheless, we argue that the main qualitative 
results, possible interpretations of the $\mathrm{C} 1$ and $\mathrm{C} 2$ phases, are robust. Moreover, the separation of the general physisorption and assembly problems should not significantly affect the results for the dilute-phase adsorption energies, the minute surface corrugation, and the enhancement of the C2 formation energy by direct $\mathrm{Bz}-\mathrm{Bz} \mathrm{vdW}$ bonding.

\section{A. van der Waals density-functional calculations}

Since details of the vdW-DF method and of the functional design are given in Refs. 19-21 and 28, we here only summarize the calculational approach. For every atomic configuration in a unit cell $V_{0}$, we obtain the self-consistent charge with traditional DFT, typically as parameterized in PBE-GGA. ${ }^{40}$ From the known electron-density variation, we also calculate a local wave vector $q_{0}(\mathbf{r})$ which characterizes the energy density in a GGA. ${ }^{20} \mathrm{Next}$, in a post-processing phase, we calculate a nonlocal-correlation term ${ }^{19}$

$$
E_{c}^{\mathrm{nl}}[n]=\frac{1}{2} \int_{V} d \mathbf{r} \int_{V_{0}} d \mathbf{r}^{\prime} n(\mathbf{r}) \phi\left(\mathbf{r}, \mathbf{r}^{\prime}\right) n\left(\mathbf{r}^{\prime}\right)
$$

as a spatial double integration over an interaction kernel $\phi\left(\mathbf{r}, \mathbf{r}^{\prime}\right)$, where the latter integration is only over the unit cell $V_{0}$. The kernel can be conveniently evaluated from a tabulated, universal form by introducing the two dimensionless variables $\Xi \equiv\left(q_{0}+q_{0}^{\prime}\right)\left|\mathbf{r}-\mathbf{r}^{\prime}\right|$ and $\xi \equiv\left(q_{0}-q_{0}^{\prime}\right) /\left(q_{0}+q_{0}^{\prime}\right)$. The nonlocal-correlation term $E_{c}^{\mathrm{nl}}[n]$ describes the interactions mediated via coupling to the electrodynamical field and allows vdW-DF to describe sparse-matter behavior. We also evaluate a (semilocal) part $E_{0}$ which is obtained from the PBE-GGA energy by adjusting the exchange to the revPBE (Ref. 41) form and the correlation to the value in the localdensity approximation. ${ }^{42}$ This term retains information about, for example, the kinetic-energy repulsion that adds to the surface corrugation. ${ }^{43}$ Finally, we determine the total vdW-DF energy as the sum

$$
E^{\mathrm{vdWDF}}[n]=E_{0}[n]+E_{c}^{\mathrm{nl}}[n]
$$

in a scheme that is transferable and capable of describing interactions for both high- and low-electron concentrations. ${ }^{21,28}$

In many respect the present vdW-DF calculations are similar to those presented in Refs. 30-33, 43, and 44. The physisorption system requires us to resolve very-small energy differences (e.g., the variation in adsorption energy with site) and to ensure accurate and well-converged evaluations of changes in the nonlocal correlation $E_{c}^{\mathrm{nl}}[n]$. Since Eq. (1) involves a real-space integral, the evaluation of $E_{c}^{\mathrm{nl}}[n]$ depends slightly on the grid sampling because of the rapid variation in the kernel at small $\Xi .{ }^{19,43}$ We employ a number of additional calculational steps to enhance accuracy and test the convergence in the evaluation of Eq. (1):

We characterize a general adsorbate system " $A$ " by the $\mathrm{Bz}$ configuration $a$ and the associated substrate configuration $S_{a}$ (which may be the frozen free surface or the surface specified by adsorbate-induced relaxations as obtained by calculations and implementations of GGA-PBE forces). For a given atomic configuration $A$, the nonlocal-correlation en- ergy (1) will be dominated by contributions from core regions at or close to the atomic positions; this causes a very small relative error $\delta E_{c}^{\mathrm{nl}}(A)$ that varies when atoms are displaced relative to the density grid. To compute adsorption energies and compare two systems, $A$ and $B$, the relevant nonlocal-correlation energy difference is instead specified by the smaller $E_{c}^{\mathrm{nl}}$ contributions that arise outside the atomic cores. However, since the two systems have different atomic configurations (and hence different alignment with the density grid), the small relative errors $\left[\delta E_{c}^{\mathrm{nl}}(A)\right.$ and $\left.\delta E_{c}^{\mathrm{nl}}(B)\right]$ need not always cancel and could affect the accuracy for systems with minute energy differences. In this study we therefore avoid direct evaluations of the nonlocal-correlation energy differences $E_{c}^{\mathrm{nl}}(A)-E_{c}^{\mathrm{nl}}(B)$ and apply, instead, the procedure described in Refs. 33 and 43. In practice, we determine $E_{c}^{\mathrm{nl}}(A)-E_{c}^{\mathrm{nl}}(B)$ in a sequence of steps where we separately calculate the $E_{c}^{\mathrm{nl}}$ changes associated with removing the $\mathrm{Bz}$ in configuration $a(b)$ to infinity, always keeping the atomic positions frozen and fixed relative to the density grid. In the vdW-DF study of the effects of adsorbate-induced relaxations of the substrate, we follow these steps by a GGAPBE calculation of the energy difference between $S_{a}$ and $S_{b}$. A previous study ${ }^{30}$ documents that a grid spacing of $0.15 \AA$ is sufficient to achieve convergence of the vdW-DF binding energy in adsorption problems when combined with the above-mentioned scheme.

The calculations of $E_{c}^{\mathrm{nl}}[n]$ via Eq. (1) are accelerated by introducing a cut-off radius $R_{\text {cut }}$ while carefully testing the convergence of the spatial double integration. Contributions of the long-range dispersive interactions to $E_{c}^{\mathrm{nl}}[n]$ fall off slower with distance $\left|\mathbf{r}-\mathbf{r}^{\prime}\right|$ for the adsorbate-slab interactions than for the $\mathrm{Bz}-\mathrm{Bz}$ interactions. The $\mathbf{r}^{\prime}$ integration in Eq. (1) exceeds the unit cell but the integrated weight of contributions outside $R_{\text {cut }}$ still decreases fairly rapidly with the value of $R_{\text {cut }} \cdot{ }^{31,44}$ We have explicitly tested that the choice $R_{\text {cut }}=23 \AA$ ensures that $E_{c}^{\mathrm{nl}}$ converges at the sub-meV level.

\section{B. Long-range indirect electronic interactions between adsorbates: nonperturbative $s$-wave treatment in the asymptotic regime}

To complete our DFT analysis we must explicitly add consistent estimates for the indirect, substrate-mediated interactions. Benzene adsorbates not only interact directly through vdW forces but also through forces mediated by the $\mathrm{Cu}(111)$ substrate. Elastic-deformation effects, ${ }^{45}$ dipolar couplings, ${ }^{46}$ and indirect electronic interactions ${ }^{6,8}$ all exhibit a power-law decay ${ }^{7,47}$ and can affect the adsorbate binding at distances $d>8 \AA$ where (we find) the direct adsorbateadsorbate vdW interaction is no longer substantial. Since we calculate the direct $\mathrm{Bz}-\mathrm{Bz}$ vdW interaction in a separate vdW-DF study that ignores the substrate, we must separately analyze the set of indirect interactions and add the relevant DFT-based terms.

Of particular interest is the surface-state-mediated indirect interaction. This metallic surface state is responsible for the dramatic images obtained with scanning-tunneling microscopy $(\mathrm{STM})^{5,36}$ Figure 2 shows that there is notable 


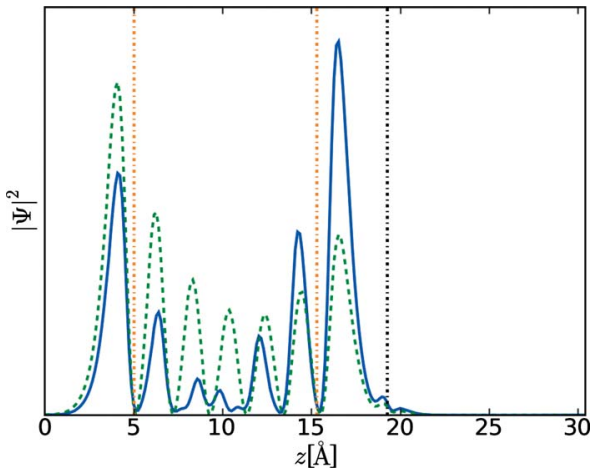

FIG. 2. (Color online) Robustness of $\mathrm{Cu}(111)$ surface-state formation to $\mathrm{Bz}$ adsorption and evidence of adsorbate-induced surfacestate scattering presented in the dilute limit with one $\mathrm{Bz}$ molecule on a frozen $3 \times 3 \mathrm{Cu}(111)$ supercell. The figure shows the variation in the in-plane average of the surface-state wave functions squared, $|\Psi|^{2}=A^{-1} \int_{2 \text { Dcell }} d A\left|\psi_{\text {surf-st. }}\right|^{2}$ (evaluated at the $\bar{\Gamma}$ point) along the surface-normal coordinate $z$ for our choice of a six-layer copper slab (with top and bottom $\mathrm{Cu}$ layers identified by light dashed lines). The physisorption position of the $\mathrm{Bz}$ adsorbates is indicated by the black dashed line (near $z=19 \AA$ ). Many layers are necessary to fully describe the surface-state properties and, in a slab geometry, to decouple the surface state on the top and on bottom sides. Our choice of six copper layers proves to be the smallest that exhibit clear signs of a Shockley surface state - an exponential decay of the surface state into the bulk and a large spill out far beyond the outer layers. The approximate symmetry of the top-side (solid curve) and bottom-side (dashed curve) surface states implies that the surface states survive the physisorption in the dilute limit. At the same time, the oscillatory behavior evident at the position of the adsorbate shows that the $\mathrm{Bz}$ interacts with surface-state electrons.

coupling between the $\mathrm{Bz}$ adsorbate and the surface-state wave function even in a dilute-coverage regime. This conclusion is expected from the analogous case of atomic adsorption $^{37}$ on $\mathrm{Cu}(111)$ (Ref. 5) where the measured adsorption-induced phase shifts are known to be large$\Theta=\Theta_{F} \sim \pi / 2$-indicating that a perturbative treatment of the surface-state-mediated adsorbate-pair interaction is inadequate. Our vdW-DF calculations of Bz physisorption suggest that the dipolar and elastic interactions between $\mathrm{Bz}$ adsorbates should be insignificant: a Bader analysis ${ }^{38,39}$ documents vanishing electron transfer and we find only very small adsorbate-induced relaxations, $\sim 30 \mathrm{meV}$, which do not change the relative stability of the various sites. However, Fig. 2 shows that the metallic surface state survives the $\mathrm{Bz}$ adsorption and therefore can mediate an interaction between adsorbates. Figure 2 also demonstrates that $\mathrm{Bz}$ affects the surface-state scattering and produces, in turn, a longrange indirect electronic interaction. ${ }^{5-8}$ The minor adsorbateinduced relaxations will only enhance the surface-state scattering by increasing the coupling between the surface-state and evanescent bulklike states around the adsorption site.

Unlike the other adsorbate-adsorbate interactions, the indirect electronic interaction is oscillatory. At short range, it depends on all the occupied states but asymptotically the behavior is dominated by the $\mathbf{q}$ state at the Fermi level whose velocity is parallel to the separation vector $\mathbf{d}$ between the adsorbates. $^{7}$ While this interaction is typically anisotropic, the metallic (Shockley) surface-state band on $\mathrm{Cu}(111)$ is circularly symmetric, so the interaction becomes isotropic, and the relevant $\mathbf{q}_{F}$ is parallel to $\mathbf{d}$. These conditions lead to the perturbative treatment well known as the Ruderman-KittelKasuya-Yosida (RKKY) interaction, ${ }^{48,49}$ which has recently been applied to actively investigated low-dimensional systems. ${ }^{50}$ A significant effect of mediation by metallic surface states is that the envelope decays exceptionally slowly $\propto d^{-2}$ rather than the usual $\propto d^{-5}$ for mediation by bulk states at metal surfaces. ${ }^{5-8}$

The primary modification due to a nonperturbative treatment is the addition of a phase shift in the argument of the sinusoidal function in the RKKY expression (and for Friedel oscillations in general). To explicate this result, we extend our regular vdW-DF calculations with a Harris-functional scheme, ${ }^{29}$ which formally constitutes an asymptotic DFT evaluation $^{8}$ of the indirect electronic interactions between $\mathrm{Bz}$ adsorbates. This choice permits a consistent description of the nonperturbative scattering and interaction effects arising with general (complex) $s$-wave surface-state phase shifts. The complex phase shift reflects transitions both among surface-state wave vectors and into evanescent bulk states. A simple $s$-wave characterization, ${ }^{8}$ with simultaneous descriptions of both scattering channels, has proven reasonably successful in estimating the resulting indirect electronic interaction for a pair of atomic adsorbates; ${ }^{5}$ the $s$-wave characterization should also be applicable for $\mathrm{Bz}$ on $\mathrm{Cu}(111)$ because the coupling of $\mathrm{Bz}$ to the substrate occurs in a relatively confined area.

The $s$-wave formulation defines an effective Fermi-level $T$ matrix $t_{0}\left(\epsilon_{F}\right) \propto\left[\exp \left(i 2 \delta_{0}\right)-1\right]$ given by a complex phase shift $\delta_{0}=\delta+i \delta^{\prime \prime}$ and formally expressing the probability for transition entirely within the surface-state channel. Unlike for a strictly two-dimensional (2D) scattering system, however, the intrasurface-state (ISS) scattering is given both an ISS phase shift $\delta$ and an ISS reflectance $r=\exp \left(-2 \delta^{\prime \prime}\right)$. The latter corresponds to a bulk-state absorption probability $1-r$, which cannot vanish since the adsorbates cause surface-state electrons to scatter into bulk states. The nonperturbative analysis leads to an effective Fermi-level phase shift $\Theta$ which can be obtained from ${ }^{8}$

$$
\begin{aligned}
\tan \left[\Theta\left(r, \delta=\delta_{F}\right)\right] & =\frac{1-r \cos (2 \delta)}{r \sin (2 \delta)} \\
\Rightarrow \Theta & \sim \frac{\pi}{2}-\frac{2 r}{1 \mp r} \varphi+\frac{4\left(r \pm r^{2}\right)}{3(1 \mp r)^{3}} \varphi^{3}+\mathcal{O}\left(\varphi^{5}\right),
\end{aligned}
$$

where the expansion is around either end point 0 or $\pi / 2$, with $\varphi=\delta$ or $(\pi / 2)-\delta$, with the upper or lower sign applying, respectively. For small $r, \Theta(r, \delta)$ is nearly symmetric about $\delta=\pi / 4$, descending linearly (from value $\pi / 2$ ), with a shallow slope, from either end point. As $r$ increases, the magnitudes of the linear slopes increase asymmetrically, with a shift of the minimum $\delta_{\text {min }}$ to progressively smaller values, while the minimum value of $\Theta, \Theta\left(r, \delta_{\min }\right)$, decreases continuously from $\pi / 2$ to 0 ; explicitly 


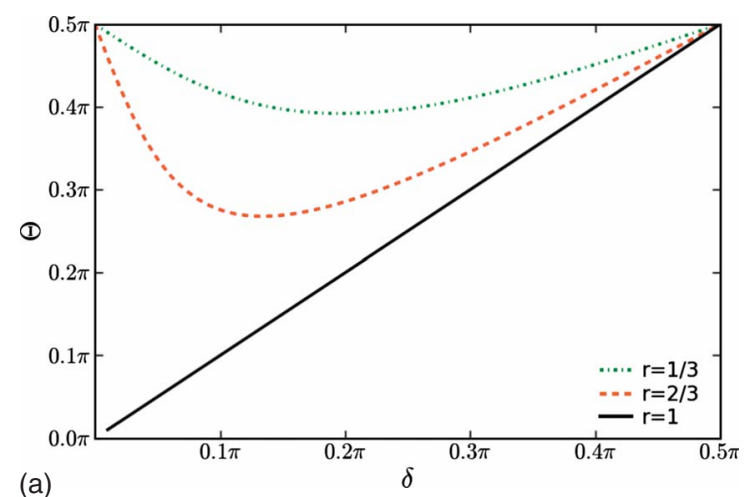

(a)

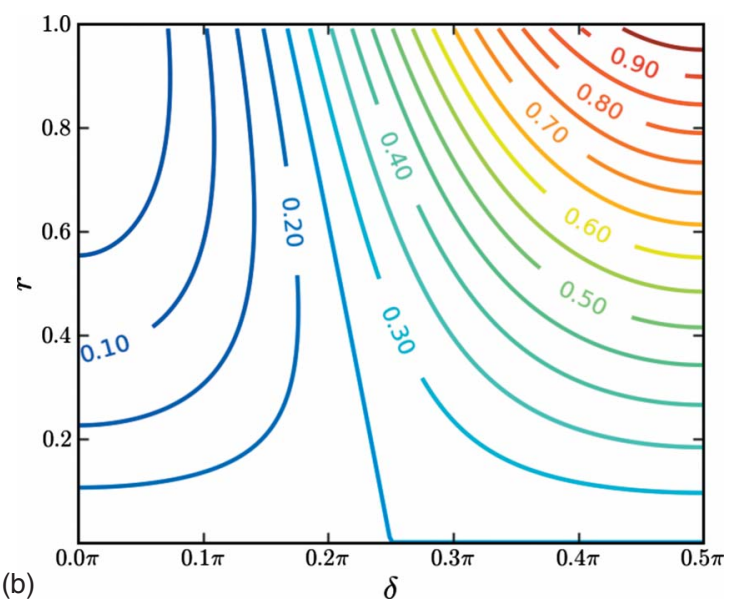

FIG. 3. (Color online) Dependence of effective interaction phase shift $\Theta$ (top panel) and scaled interaction strength $A$ of indirect electronic adsorbate-pair interaction [Eq. (5)] with detailed nature of adsorbate-induced $s$-wave scattering of surface-state electrons. The variation is expressed by the phase shift for intrasurface-state transitions $\delta$ and the intrasurface-state reflectance $r$ (where 1-r describe the probability that the adsorbate causes a scattering from a surface state into an evanescent bulklike state). Analogy with the case of interactions between $\mathrm{Cu}(111)$ adatoms suggests that it reasonable to expect both $1-r>0$ and $\delta>0$. While we do not have explicit determinations of the relevant parameters for $\mathrm{Bz}$ on $\mathrm{Cu}(111)$, the panels suggest that values, $\Theta>\pi / 3$ and $A>0.1$, can be considered typical for indirect electronic adsorbate-pair interactions [valid for the present case of $\mathrm{Bz}$ on $\mathrm{Cu}(111)$ as well as for $\mathrm{Cu}$ adatoms on $\mathrm{Cu}(111)$ (Ref. 5)].

$$
\delta_{\min }=\frac{1}{2} \cos ^{-1} r, \quad \Theta\left(r, \delta_{\min }\right)=\tan ^{-1} \frac{\sqrt{1-r^{2}}}{r} .
$$

The upper panel of Fig. 3 depicts the relation between $\Theta$ and $\delta$ for three representative $r$ values. We determine $E_{\text {pair }}^{\text {ans }}(d)$, where the superscript denotes that this is the asymptotic form of the indirect electronic adsorbate-pair interactions, computed with the nonperturbative formal Harris functional for $s$-wave scattering, through the analytical evaluation ${ }^{5,7}$

$$
E_{\text {pair }}^{\text {ans }}(d)=-A(r, \delta)\left(\frac{4 \epsilon_{F}}{\pi^{2}}\right) \frac{\sin \left[2 q_{F} d+2 \Theta(r, \delta)\right]}{\left(q_{F} d\right)^{2}} .
$$

Here

$$
A(r, \delta)=\left[(1-r)^{2} / 4+r \sin ^{2}(2 \delta)\right]
$$

represents a scaled, dimensionless interaction strength, 0 $\leq A \leq 1$. For $\mathrm{Cu}(111)$ the wave vector $q_{F}$ of the surface state is $0.22 \AA^{-1}$ and the Fermi energy $\epsilon_{F}$ is $0.39 \mathrm{eV}^{4}$ The explicit values of $\delta$ and of $r$, or equivalently $\Theta$ and $A$, depend on the details of the Bz-induced scattering, for which we have no experimental characterization; we stress that, in the present $s$-wave analysis, the effective interaction phase is the same $\Theta$ that characterizes the STM measurements of the local density-of-state variation. .,36 $^{2}$

Figure 3 displays the possible variation in interaction phase $\Theta$ (upper panel) and scaled interaction strength $A$ (lower panel) with the $s$-wave modeling parameters $\delta$ and $r$. The perturbative regime of RKKY corresponds to the limit $(r \rightarrow 1, \delta \rightarrow 0)$ while $r \rightarrow 0$ corresponds to the case of completely absorbing scattering (all surface-state electrons scatter into the bulk), for which Eqs. (4) and (6) indicate $\Theta$ $=\pi / 2$ and $A=0.25$, respectively. In making these plots we have implicitly assumed a positive interaction phase shift $\Theta>0$ (as was measured for atomic adsorbates ${ }^{5}$ ). We expect $\mathrm{Bz}$ adsorbates to produce significant coupling between surface and bulk states, $r \neq 1$ and finite ISS scattering $\delta \neq 0$. The lower panel of Fig. 3 shows a contour plot of the scaled interaction strength $A$; even moderate values of the ISS scattering phase shift $\delta$ and of the bulk-state absorption, $1-r$, produces significant values of the interacting strength. Generic values for the ISS scattering parameters ( $\delta$ and $r$ ) produce an interaction strength and phase which are comparable to or larger than those characterizing interactions between $\mathrm{Cu}$ adatoms on $\mathrm{Cu}(111)$ (Ref. 5): $A_{\mathrm{Cu} / \mathrm{Cu}}=0.08$ and $\Theta_{\mathrm{Cu} / \mathrm{Cu}}=\pi / 3$.

It is interesting to compare the indirect electronic interactions between pairs of $\mathrm{Bz}$ and pairs of $\mathrm{Cu}$ adatoms on $\mathrm{Cu}(111)$. In both cases is there is essentially no charge transfer. The Friedel sum rule ${ }^{51}$ then tells us ${ }^{8}$ that the coupling between surface and bulklike states must be the primary cause of the Friedel oscillations. On the one hand, since Bz is physisorbed to $\mathrm{Cu}(111)$, its impact should be smaller than chemisorbed $\mathrm{Cu}$ adatoms, with a smaller adsorbate-induced coupling between the surface and bulk states.,36 On the other hand, Bz has a quadrupole moment, defined by a total of six $\mathrm{C}-\mathrm{H}$ bonds with a nonzero dipolar moment that will scatter the surface-state electrons. In a simple tight-binding model of weak adsorption, the indirect interaction varies as the square of the adsorption energy. ${ }^{7}$ It is plausible that scattering off each carbon atom in $\mathrm{Bz}$ produces an indirect electronic pair interaction with a nonvanishing interacting strength and phase so that there is a significant (though likely not 36-fold) enhancement of the individual interactions. One might instead view the adsorption as due to a molecular orbital of each cyclic group, implying that the indirect interactions of anthracene pairs might be 3-9 times those of benzene at the same separation. Lukas et $a .^{12}$ did find the intriguing result that the binding energies of $\mathrm{C}_{4 n+2} \mathrm{H}_{2 n+4}$ on $\mathrm{Cu}(111)$ for $n=0,1,2,3$ (acetylene, benzene, naphthalene, and anthracene) are approximately collinear as a function of $n$ with the binding energy of anthracene about five times that of acetylene and somewhat more than twice that of benzene. In summary, it is not clear how several adsorbates within the 
screening length of the bulk electrons couple to the surface state compared to just a single adatom. This issue begs further investigation.

\section{Adsorption and surface assembly energies}

The adsorption energy is given by the difference between the total energy for a given configuration and the total energy of the $\mathrm{Bz}$ and the surface in isolation

$$
E_{\mathrm{ads}}^{\mathrm{vdWDF}}=E^{\mathrm{vdWDF}}(h)-E^{\mathrm{vdWDF}}(h \rightarrow \infty) .
$$

[In practice, due to the finite size of the unit cell, each term of the RHS of Eq. (7) contains $\sim 30 \mathrm{meV}$ per $\mathrm{Bz}$ due to lateral vdW interactions, which essentially cancel after the subtraction.] The full formation energy when including the energy gained by forming an overlayer is given by the sum of the adsorption energy and the 2D assembly energy $E_{\text {form }}$

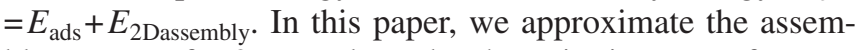
bly energy of a $2 \mathrm{D}$ overlayer by the pairwise sum of $\mathrm{Bz}-\mathrm{Bz}$ interactions:

$$
E_{2 \text { Dassembly }}^{\text {app }} \approx \frac{1}{2} \sum_{i \neq j} E_{\text {pair }}\left(\mathbf{r}_{i j}\right) .
$$

This corresponds to neglecting higher-order (multimolecule) $\mathrm{Bz}-\mathrm{Bz}$ interactions both for the van der Waals and indirect electronic interactions. A test calculation with a moderately dense benzene overlayer indicates that changes in the vdW-DF result can alter the formation energy by a few tens of meV. For indirect electronic interactions at large separations, the contribution of trio (three-adatom, nonpairwise) interactions decays only slightly faster than pair interactions and should be, very roughly, 1/4 the magnitude. ${ }^{8,9}$ While such higher-order interactions are needed to achieve high accuracy, they are not essential for our analysis. We further argue that to a good approximation elastic effects and surface corrugation can be neglected in computing $E_{\text {form }}$. We thus calculate the direct component of the $\mathrm{Bz}-\mathrm{Bz}$ interaction in isolation from the substrate: $E_{\mathrm{pair}, h \rightarrow \infty}^{\mathrm{vdWDF}}$. Again this energy is the difference between nearby molecules and widely separated ones: $E_{\text {pair }}^{\mathrm{vdWF}}(d)=E^{\mathrm{vdWDF}}(d)-E^{\mathrm{vdWDF}}(d \rightarrow \infty)$. The interactions between molecules mediated by the surface, formally included in $E_{0}$, is reintroduced by including the leading-order asymptotic part of the surface-state-mediated indirect interaction as discussed in the previous section

$$
E_{\text {pair }} \approx E_{\text {pair }, h \rightarrow \infty}^{\mathrm{vdWDF}}+E_{\text {pair }}^{\mathrm{ans}}
$$

The asymptotic expression $E_{\text {pair }}^{\text {ans }}$ is expected to provide a good approximation to the full-pair indirect interaction in the regime where the latter dominates the direct vdW interaction.

\section{Choice of unit cell, computational details}

The self-consistent charge density is found using the plane-wave and ultrasoft-pseudopotentials code DACAPO (Ref. 52) with GGA in the PBE flavor. For the physisorption study on a $3 \times 3$ slab unit cell with six copper layers, we use a Monkhorst-Pack $(3,3,1)$ k-point sampling. The study of direct $\mathrm{vdW}$ interaction between $\mathrm{Bz}$ pairs in the dilute case was done in a very-large unit retaining only the $\Gamma$ point. The plane-wave energy cutoff was set higher than $500 \mathrm{eV}$ to ensure that the spatial gridding was consistently better than $0.14 \AA$.

We place a Bz molecule on a $3 \times 3 \mathrm{Cu}(111)$ supercell with a slab containing about six copper layers. The choice of insurface dimensions corresponds to a Bz-to-Bz separation of $a_{\text {cell }}=7.74 \AA$. Our system is large enough to extract the essential physics but not so large as to preclude doing so in a timely fashion. Our vdW-DF calculations are based on a periodic unit cell and the choice of supercell does leave a small (but fixed) residual molecule coupling arising from both direct intermolecular vdW interactions and from substratemediated interactions. The vdW-DF study of the direct vdW interaction between $\mathrm{Bz}$ pairs indicates that the effect of direct $\mathrm{vdW}$ bonding is small (three times $\sim 10 \mathrm{meV}$ ); this energy is assumed to cancel in the subtraction in Eq. (7). However, the long-ranged (and oscillatory) nature of the indirect electronic interaction motivates a separate discussion as do the convergence of physisorption energies with slab thickness.

We note that the effects of indirect electronic adsorbateadsorbate interaction will be contained in very-large-scale DFT calculations ${ }^{10}$ and that vdW-DF would formally contain such a contribution, primarily in the semilocal term $E_{0}$. However, our specific choice of supercell with six copper layers does not allow more than a qualitative discussion of the surface state, Fig. 2, and it is not possible to use our vdW-DF calculations for a quantitative analysis. Instead we note, based on an assumption of completely absorbing surfacestate scattering in the asymptotic limit, that the magnitude of the fixed contribution from indirect electronic interactions (arising from our use of an implicit periodicity in our vdW-DF unit cell) can be approximated as $3 \times 2=6 \mathrm{meV}$ [according to Eq. (5), which is not fully valid at this notquite-asymptotic separation]. This value is much smaller than the calculated vdW-DF value for the Bz-physisorption energies and we choose not to correct for the fixed effect of implicit indirect electronic interaction in presenting our $\mathrm{vdW}$-DF results for the isolated-molecule adsorption. It is safe to assume that these inter-unit-cell contributions will not modify the vdW-DF results of extremely low diffusion barriers and essentially corrugation-free dynamics of individual Bz adsorbates.

A partially related issue is convergence of vdW-DF energy differences with slab thickness. We find the variation in the physisorption energy with adsorption site to be very small, a finding that motivates us to enhance the accuracy in the vdW-DF calculations. To test convergence with respect to slab thickness, we compared the results of vdW-DF calculations for two different adsorbate geometries. Specifically, we have determined how many $\mathrm{Cu}$ layers $n$ are needed to accurately and reproducibly predict energy differences for adsorption configurations FCC- $I$ and FCC-R30 $\Delta E_{n}^{\mathrm{vdWDF}}$ $=E_{\mathrm{FCC}-\mathrm{I}, n}^{\mathrm{vdWDF}}-E_{\mathrm{FCC}-\mathrm{R} 30, n}^{\mathrm{vdWDF}}$. Here, $\Delta E_{n}^{\mathrm{vdWDF}}$ changed from $10 \%$ when comparing four and five layers to $2 \%$ when comparing six and seven layers. We thus conclude that six copper layers give satisfactory convergence. The somewhat slow convergence might be related to the need for several $\mathrm{Cu}$ layers before a clear signature of the Shockley surface begins to emerge. 

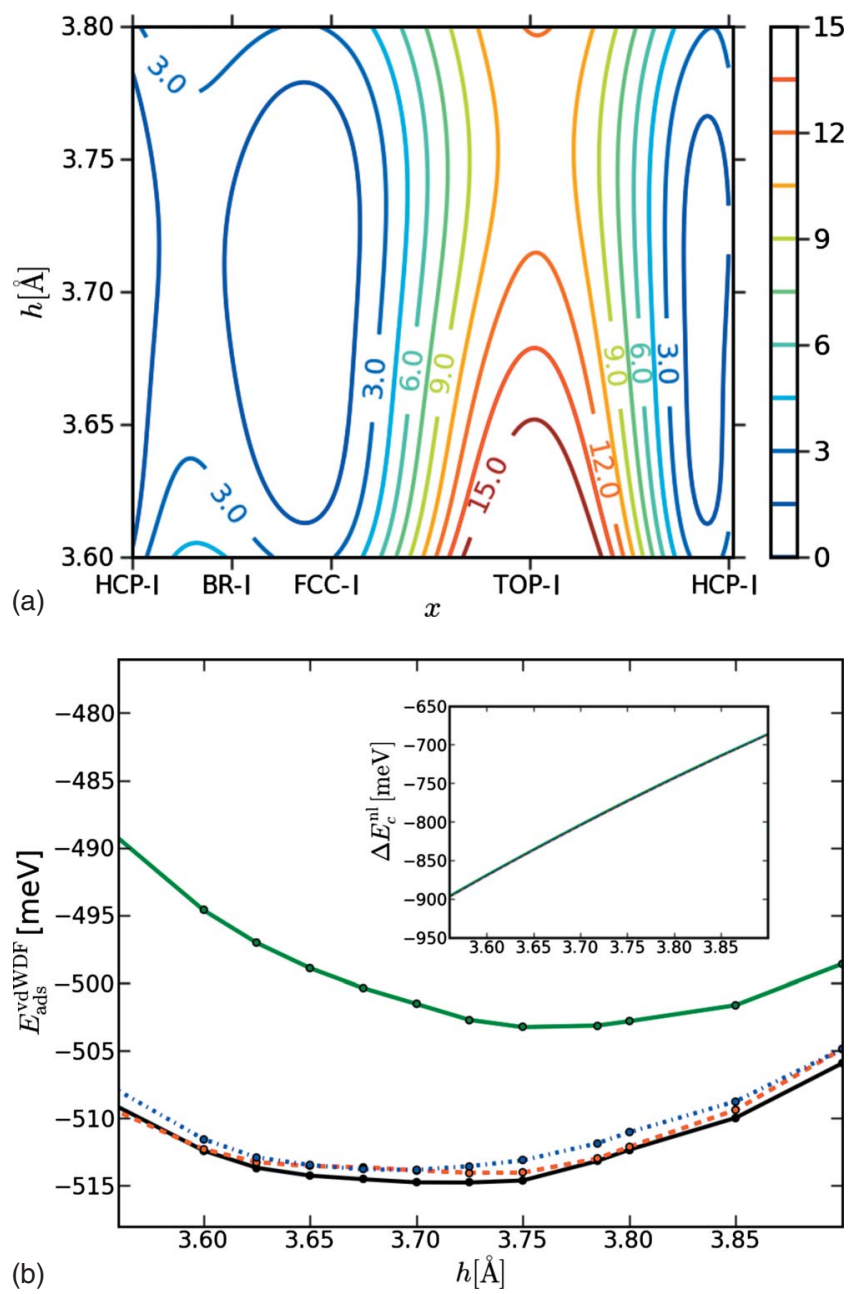

FIG. 4. (Color online) Potential-energy curves for $\mathrm{Bz}$ on $\mathrm{Cu}(111)$, for computing diffusion barrier. For the contour plot shown in the upper panel, the molecule is allowed to change its separation from the surface at each position. The vertical axis gives the separation from the surface and the horizontal axis shows the translation along the arrow indicated in Fig. 1. The bar to the right shows the energy scale in $\mathrm{meV}$ for the equipotential lines. This plot was smoothed using spline interpolation. The lower panel shows the potential-energy curves for $\mathrm{Bz}$ adsorption in the four highsymmetry sites with $I$ orientation. The upper lower (upper) solid curve is for the FCC-I (TOP-I) site, the dashed (dotted) is for the HCP-I (BR-I) site. The inset in the lower panel shows the nonlocal part of the potential-energy curves, which are very similar, illustrating that the energy difference between the sites stems primarily from $E_{0}(n)$.

\section{SINGLE-MOLECULAR ADSORPTION AND DIFFUSION}

\section{A. Physisorption energy}

Figure 4 displays the potential-energy landscape, found by performing a potential-energy search along an axis of high-symmetry sites, as shown in Fig. 1, for both BZ orientations (configurations) $I$ and $R 30^{\circ}$, as defined in the caption of that figure. We allow the molecule to adjust its height at each step, but do not allow further substrate relaxation. The spline-interpolated result for the $I$ configurations are shown
TABLE I. Interaction energies for adsorption of benzene $\left(\mathrm{C}_{6} \mathrm{H}_{6}\right)$ at various positions on $\mathrm{Cu}(111)$ in order of decreasing stability, with $\mathrm{vdW}-\mathrm{DF}$ but frozen substrate, with vdW-DF and relaxation, and without vdW-DF (from Ref. 27).

\begin{tabular}{lccc}
\hline \hline Site & $\begin{array}{c}E_{\mathrm{ads}}^{\mathrm{vdW}-\mathrm{DF}} \\
\mathrm{meV}\end{array}$ & $\begin{array}{c}E_{\mathrm{relax} \text { ads }}^{\mathrm{vdW}} \\
\mathrm{meV}\end{array}$ & $\begin{array}{c}E_{\mathrm{ads}}^{\mathrm{GGA}} \\
\mathrm{meV}\end{array}$ \\
\hline FCC-I & -515 & -548 & -23 \\
HCP-I & -514 & -548 & -26 \\
BR- $I$ & -514 & -547 & -16 \\
TOP-I & -503 & -533 & 44 \\
HCP- $R 30^{\circ}$ & -499 & -533 & -18 \\
FCC- $R 30^{\circ}$ & -498 & -527 & -17 \\
BR- $R 30^{\circ}$ & -497 & -529 & -21 \\
TOP- $R 30^{\circ}$ & -490 & -520 & 10 \\
\hline \hline
\end{tabular}

in the upper panel, while the lower panel shows the binding curve for four of the high-symmetry sites. All I configurations have a lower energy than all $R 30^{\circ}$ configurations, and the line between HCP-I, BR-I, and FCC-I sites is found to be almost degenerate, with a predicted adsorption energy of $0.51 \mathrm{eV}$, where FCC-I is slightly more favorable than the two other sites. ${ }^{53}$

The good agreement with the experimental binding energy $0.6 \mathrm{eV}$ (Refs. 12 and 13) further demonstrates that the use of the vdW-DF offers a viable way to describe the binding between organic molecules and noble-metal surfaces, even in the presence of a metallic surface state. The accuracy of using vdW-DF for this system is similar to its performance in general. The accuracy is also close to that found for $n$ butane on $\mathrm{Cu}(111) .^{21}$

Our results clearly demonstrate the nonlocal nature of the binding. The binding separation of $3.75 \AA$ is a typical separation distance for physisorption; furthermore, with a Bader analysis ${ }^{38,39}$ we found vanishing charge transfer between the surface and the $\mathrm{Bz}$ molecule.

In the results presented so far, we use a frozen substrate, thereby neglecting elastic effects. To assess the importance of these effects, we perform for every high-symmetry site a set of separate calculations, in which we let the top four layers of the surface relax and fix the coordinates of the benzene atoms in positions ranging from 0.1 above to $0.1 \AA$ below the optimal separations to the unrelaxed surfaces. We use PBE-GGA for this relaxation, while in the postprocessing phase, the relaxation energy is estimated from vdW-DF.

Table I give the adsorption energy of the eight highsymmetry sites, for a frozen substrate, a substrate allowed to relaxed and for the purely PW91-GGA calculation reported in Ref. 27. It shows that allowing relaxation lowers the adsorption energy within vdW-DF by between 29 and $34 \mathrm{meV}$, getting even closer to the experimental value of $0.6 \mathrm{eV}$. Allowing relaxation gives only minor corrections to the relative favorability of the different adsorption sites. Note, though, that the calculations with relaxation are more sensitive to the shifting from the PBE to the revPBE flavor of exchange in the post-processing phase and so are converged only at the 
2-3 meV level rather than the sub-meV convergence of the calculations without adsorption-induced relaxation. Since the better convergence gives us greater confidence in the computations with a frozen substrate, we base our investigation of the surface-corrugation results on them.

Finally, Table I shows dramatically the need for including vdW interactions to accurately describe this adsorption system. Calculations which do not include vdW [in this case ${ }^{27}$ but use PW91-GGA done with the Vienna Ab-initio Simulation Package (VASP)] underestimate the adsorption energy by a factor of around 20. For the two TOP configurations, the benzene is not even bound. The near degeneracy of the three lowest configurations is lost and the general ordering is considerably different. The theme of the overall favoring of $I$ configurations is absent. Thus, such calculations are inadequate for semiquantitative and perhaps even qualitative purposes.

\section{B. Surface corrugation, barrierless diffusion on a honeycomb lattice}

Figure 4 presents our vdW-DF results for diffusion barriers and shows that $\mathrm{Bz}$ adsorbates are expected to optimize their positions relative to their mutual interactions with the substrate corrugation potential playing a relatively minor role. Table I presents an overview of the surface corrugation as calculated in vdW-DF and GGA. We note that the surface corrugation (and hence diffusion barriers) is at least an order of magnitude larger in GGA but we do not believe that the GGA finding, top sites being unstable toward desorption, is physical. The vdW-DF calculations predict a much smaller corrugation: a low-temperature preference for placing $\mathrm{Bz}$ adsorbates around the nearly degenerate FCC-I, HCP-I, and $\mathrm{BR}-I$ sites with a relative cost of around $11 \mathrm{meV}$ for placing $\mathrm{Bz}$ on a TOP-I site. Our vdW-DF description may slightly underestimate the corrugation because it is likely to weakly overestimate the physisorption height $h$. The TOP-I versus FCC- $I /$ HCP-I/BR-I energy difference is in any case sufficient to affect the expected equilibrium distribution at $77 \mathrm{~K}$. Our vdW-DF results shows that the Bz adsorbates at low temperatures will concentrate in a honeycomb pattern (physisorption valley) formed by linking the FCC-I, HCP-I, and BR-I sites.

At the same time, we find that the $\mathrm{Bz}$ adsorbates effectively experience no diffusion barriers and are free to respond to their mutual interaction. All of the diffusion barriers are tiny compared to those for chemisorbed atoms; for a $\mathrm{Cu}(111)$ adatom the experimental and theory results for the diffusion barrier is $40 \mathrm{meV} .^{5,22-24}$ Even for a hypothetical diffusion path, HCP- $I \rightarrow$ TOP- $I \rightarrow$ FCC- $I$, the peak of the barrier $(11 \mathrm{meV})$ is small enough to allow a diffusion rate larger than $1 \mathrm{~s}^{-1}$ at $T>4 \mathrm{~K}$. The diffusion barrier for motion within the honeycomb pattern, along the path HCP-I $\rightarrow$ BR- $I \rightarrow$ FCC- $I$, is less than $1 \mathrm{meV}$ and we stress that the $\mathrm{Bz}$ adsorbates can move continuously across the surface, using this bendlike type of diffusion. Again, our vdW-DF calculations may slightly underestimate the diffusion barriers but it is clear that the $\mathrm{Bz}$ adsorbates are free to respond to their mutual interactions on the $\mathrm{Cu}(111)$ surface.
TABLE II. Binding difference between $R 30^{\circ}$ sites and $I$ sites, which also give the rotational barriers. The second column shows the results for a frozen substrate while the third column gives the results when relaxation is included.

\begin{tabular}{lcc}
\hline \hline Site & $\begin{array}{c}\Delta E_{\text {ads }}^{\mathrm{vdWDF}} \\
\mathrm{meV}\end{array}$ & $\begin{array}{c}\Delta E_{\text {relax ads }}^{\mathrm{vdWDF}} \\
\text { meV }\end{array}$ \\
\hline hcp & 15 & 15 \\
BR & 17 & 18 \\
fcc & 17 & 21 \\
TOP & 13 & 13 \\
\hline \hline
\end{tabular}

Despite such low barriers, there are many reports of lattice-gas phases of physisorbed atoms at submonolayer coverages. ${ }^{54}$ For example, while there is an incommensurate phase of $\mathrm{Kr}$ on graphite, there is also a widely studied commensurate phase, $(\sqrt{3} \times \sqrt{3}) R 30^{\circ}$, with one in three sites occupied on the hexagonal grid formed by the centers of the honeycombs. ${ }^{55}$ The theoretical analyses ${ }^{56}$ of the disordering phase transition involves a lattice-gas model appropriate to adsorption on a grid of sites. Likewise, we find such behavior below for the two condensed phases of $\mathrm{Bz}$ on $\mathrm{Cu}(111)$.

\section{Rotational barriers}

Having established that the benzene can move almost freely on the surface of $\mathrm{Cu}(111)$, we investigate whether it can also rotate freely. The symmetry of the $I$ and $R 30^{\circ}$ configurations and the monotonic increase in the barrier profile from HCP-I/FCC-I to TOP-I allow us to assume that the peak of the rotation barrier is given simply by the energy difference between configuration $I$ and configuration $R 30^{\circ}$.

Table II compares the energy differences between $\mathrm{Bz}$ adsorbates in orientation $R 30^{\circ}$ and $I$, which in turn determine the rotational barriers. The vdW-DF results show that individual molecules can easily rotate, even though the associated activation energy is slightly higher than for diffusion.

\section{SURFACE ASSEMBLY}

The vdW-DF findings of small diffusion barrier and lack of expected surface corrugation for individual $\mathrm{Bz}$ adsorbates support the picture of an essentially free 2D gas of $\mathrm{Bz}$ molecules in the dilute regime. This is what was found on $\mathrm{Cu}$ terraces at $T=77 \mathrm{~K} .{ }^{16,17}$ Further, the finding that the location of $\mathrm{Bz}$ adsorbate to some extent decouple from the underlying copper lattice, leaves the stage open for other phenomena to dominate the interplay between molecules at higher coverages and determine the $2 \mathrm{D}$ surface assembly of the $\mathrm{Bz}$ adsorbates.

Dougherty et al. $^{15}$ observed the coexistence of a highdensity $\left(2.54 \mathrm{~nm}^{-2}\right)$ phase C2 and a low-density $\left(1.10 \mathrm{~nm}^{-2}\right)$ phase $\mathrm{C} 1$, both forming hexagonal patterns. We seek insight and provide an analysis in which we first calculate the direct and indirect $\mathrm{Bz}-\mathrm{Bz}$ interactions and then combine these into an effective interaction potential. We finally use this interaction potential to characterize the high-density phase and discuss the possibility of surface-state-mediated 
TABLE III. Energy and structure of the different phases. The formation energies [calculated from the expression for $E_{\text {form }}$ after Eq. (7)] are listed both as obtained for a frozen surface and (in parentheses) in the presence of adsorbate-induced surface relaxations. The rotational barriers are given by the site-orientation dependency of the benzene molecule. For $\mathrm{C} 1$ and $\mathrm{C} 2$ data the molecules are assumed to be in the $I$ orientation with the effect of indirect electronic interactions calculated for $A=0.25$ and $\Theta=\pi / 2$ (corresponding to a choice of completely absorbing scattering). The square brackets give the values with $A=0$. The rotational excitation is calculated under the assumption that the $I-R 30^{\circ}$ interaction is limited to neighboring molecules.

\begin{tabular}{lccc}
\hline \hline & $\mathrm{C} 2$ & $\mathrm{C} 1$ & Dilute \\
\hline Formation energy (meV) & $618(-)$ & $539(572)$ & $515(548)$ \\
Expt. adsorption energy (meV) & & & $600^{\mathrm{a}}$ \\
Lattice parameter $(\AA)$ & 6.9 & 10.2 & \\
Expt. lattice parameter $(\AA)$ & $6.8^{\mathrm{b}}$ & $10.2^{\mathrm{b}}$ & \\
Rotation barrier (meV) & & $\sim 17$ & 17 \\
Rotation excitation $(\mathrm{meV})$ & $\sim 9$ & & \\
2D-assembly energy (meV) & $\sim 79[97]$ & $\sim 24$ & \\
\hline \hline
\end{tabular}

a Based on Ref. 12.

${ }^{\mathrm{b}}$ Based on Ref. 15.

forces being responsible for the structure of the low-density phase.

Table III summarizes the properties of the three $\mathrm{Bz}$ on $\mathrm{Cu}(111)$ regimes. These results can be compared with measurements to test the accuracy and predictive power of our vdW-DF calculations and the analysis of the indirect electronic interaction mediated by the $\mathrm{Cu}(111)$ surface state..$^{8,29}$

\section{A. Results for pair interactions}

There are two important forces between the molecules for the interacting system formed when multiple $\mathrm{Bz}$ adsorbates assemble on $\mathrm{Cu}(111)$. First is the direct vdW interaction between the $\mathrm{Bz}$ molecules; this interaction is medium range. Second is the indirect electronic Bz-Bz interaction, mediated by the surface-state electrons, which is long range and oscillatory. ${ }^{5-7}$ In Sec. IV A, we address the influence of these effects for pair interactions and construct an effective pair potential which can be used to discuss island formation and phase condensation of $\mathrm{Bz}$ on $\mathrm{Cu}(111) .{ }^{15}$

Figure 5 shows the vdW-DF binding curve of $\mathrm{Bz}$ pairs, $E_{\mathrm{pair}, h \rightarrow \infty}^{\mathrm{vdWD}}(d)$ for different high-symmetry configurations identified in the set of inserts and calculated in vdW-DF ignoring contact with the surface. The two Bz molecules lies entirely within a common plane. For lack of better notation in the literature, we label them acene-like $(\mathrm{A})$, phenyl-like $(\mathrm{P})$, and mixed $(\mathrm{M})$. In the $\mathrm{A}(\mathrm{P})$ configuration the displacement vector from one $\mathrm{Bz}$ to the other goes perpendicularly through an edge (a vertex) of both carbon hexagons. In the $\mathrm{M}$ configuration the displacement vector goes through a vertex of one $\mathrm{Bz}$ and an edge of the other. The binding energy is largest for

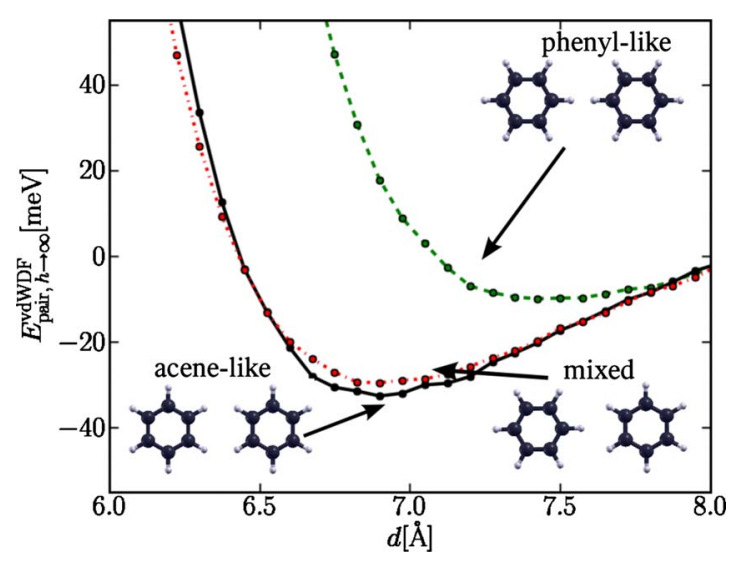

FIG. 5. (Color online) Binding curve of two Bz molecules in different high-symmetry configurations identified by the set of inserts and calculated in vdW-DF in isolation from the surface. The two Bz's lies entirely within a common plane and the abscissa gives the distance between the center of two Bz's. We label the configurations shown in the lower left, lower right and upper right corner as $\mathrm{A}, \mathrm{M}$, and $\mathrm{P}$. The most favorable A configuration (solid curve) has an optimal binding distance of $6.9 \AA$ and corresponding vdW-DF binding energy is $32 \mathrm{meV}$.

the acene-like configuration, closely followed by the mixed, with the minimum of the bonding curve at $-32 \mathrm{meV}$. In the $\mathrm{P}$ configurations bonding is much weaker and at a larger separation. The direct vdW binding clearly has a larger magnitude than the barrier profile for the adsorbed molecules. Thus, the Bz-Bz pair interactions should dominate over surface-corrugation effects at smaller separations $(d$ smaller than $\sim 8 \AA$ ). Summing the contributions of pairwise vdW bindings in A configurations, we estimate the full vdWassembly energy of an isolated sheet of $\mathrm{Bz}$ molecules in a hexagonal lattice to be $97 \mathrm{meV}$.

On the $\mathrm{Cu}(111)$ substrate, the $I$ configurations are optimal for single-molecular adsorption and the A configurations are optimal for pairs. It is thus relevant to discuss an effective adsorbate-pair interaction $E_{\text {pair }}$ with implicit assumptions of favorable molecular orientations (with both $\mathrm{Bz}$ molecules insurface orientation $I$ and with the direct $\mathrm{vdW}$ interaction specified by the A configuration). This effective (pair) potential determines adsorbate organization into a well-ordered triangular lattice structures at very low temperatures.

Figure 6 shows our result for the effective (favorablecondition) potential $E_{\text {pair }}$ with a binding curve that includes the asymptotic surface-mediated interactions given by Eq. (5). Since we lack explicit information about the detailed nature of surface-state scattering by $\mathrm{Bz}$ adsorbate, we must make assumptions for it by analogy with the case of atomic adsorption on $\mathrm{Cu}(111) .^{5}$

We give the results for the case of a completely absorbing scatterer with $A=0.25$ and $\Theta=\pi / 2$, and, for comparison, the values fitted to the experimental scattering of copper adsorbates ${ }^{5}$ with $A=0.08$ and $\Theta=0.3 \pi$. The black dotted vertical line in the figure separates the region in which van der Waals forces are important from the regions where the surface-mediated interactions dominate. For the latter region 


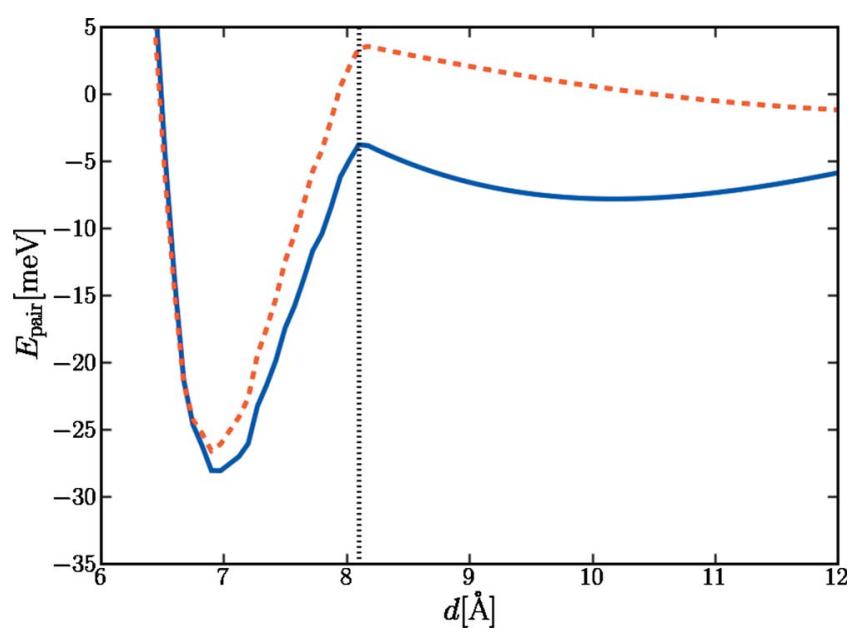

FIG. 6. (Color online) Effective binding potential of a Bz pair from the combinations of direct $\mathrm{vdW}$ and indirect electronic (surface-state mediated) $\mathrm{Bz}-\mathrm{Bz}$ interactions, assuming favorable orientations of $\mathrm{Bz}$ molecules. The upper dashed curve shows the results for the values of $A=0.08$ and $\Theta=0.3 \pi$ while the lower solid curve shows the results for $A=0.25$ and $\Theta=\pi / 2$. For $d>8.1 \AA$ (indicated by the dotted black line), the vdW interaction is negligible, and the displayed interaction is given by the formal Harrisfunctional evaluation, Eq. (5).

only the indirect electronic interactions are included. For $A$ $=0.25$, the curve has two minima, the first stemming from direct intermolecular interactions, and the second, weaker one stemming from the sinusoidal form of the contribution Eq. (5). For $A=0.08$, the indirect electronic interaction only creates a second, very weak minimum $(1.5 \mathrm{meV})$ at $14 \AA$, but do still create an energy barrier for the adsorbates trying to move from the $\mathrm{C} 1$ phase and assemble into the vdWbound $\mathrm{C} 2$ phase.

\section{B. Van der Waals lattice (C2)}

The optimal pair separation of $6.9 \AA$, as shown in Figs. 5 and 6 , illustrates that it can be favorable for islands to form at benzene separations somewhat shorter than that of the 3 $\times 3$ unit cell: $3 a \approx 7.74 \AA$. The primary limitation for the density of these islands is the kinetic-energy repulsion between benzene molecules. Assuming that the molecules lie flat on the copper surface and form a hexagonal pattern with pairs of molecules in A configurations, we estimate a density of $2.42 \mathrm{~nm}^{-2}$, very close to the experimentally determined density.

Figure 7 illustrates a curious consequence for the adsorbate dynamics in phase $\mathrm{C} 2$. We include it as the phenomenon is a consequence of the proposed interpretation of phase C2 as a planar hexagonal pattern of $\mathrm{Bz}$ adsorbates, primarily organized in orientation $I$, with pairs of Bz's in the A configuration and assembled by direct vdW bonding; an experimental observation of this consequence would constitute a specific test of the proposed interpretation of phase $\mathrm{C} 2$ as a
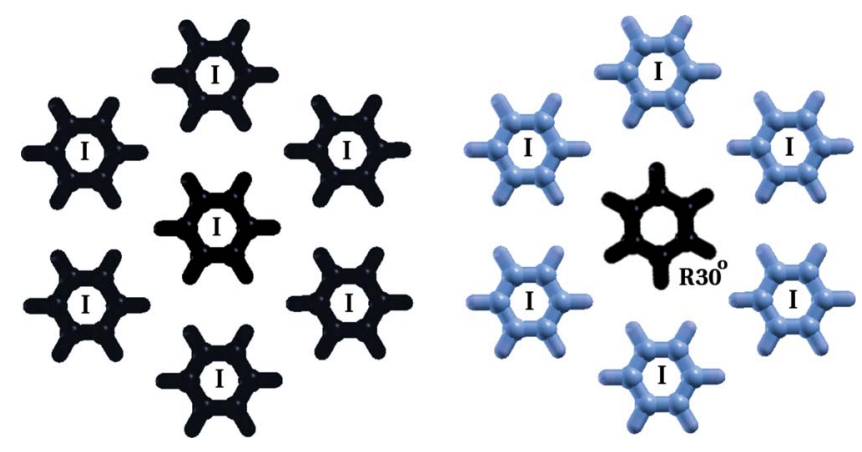

FIG. 7. (Color online) Schematic illustration of a rotational excitation in the hexagonal phase $\mathrm{C} 2$ and of the interlocking effects which (at low temperatures) will prevent neighboring adsorbates from rotating until the original rotational excitation relaxes. The figure shows two near-degenerate configurations of benzene on $\mathrm{Cu}(111)$, in the left panel all molecules are in the ground-state packing with all adsorbates in the $I$ orientation while in the right picture one of molecules has switched to the $R 30^{\circ}$ orientation. This switch corresponds to six adsorbate pairs changing to the $\mathrm{M}$ configuration. For pairs, this switch corresponds to six of them changing to the $\mathrm{M}$ configurations. While this rotational excitation does not cost much energy per se, it does block the rotation of all the neighboring molecules [marked in light blue (gray in print)] until the center molecules relaxes back to orientation I.

vdW-bound assembly. Based on vdW-DF calculations of the direct bonding, we find that the hexagonal pattern will permit a low-energy excitation with a rotation of a single $\mathrm{Bz}$ adsorbate from orientation $I$ to $R 30^{\circ}$. This switch corresponds to six $\mathrm{Bz}$ pair configurations changing from A to $\mathrm{M}$. However, as illustrated in the right panel, such a rotational excitation will (at low temperature) cause a blocking of rotations for all neighboring $\mathrm{Bz}$ adsorbates. This effect is due to the strong repulsion at the typical $\mathrm{C} 2$ separation for pairs of Bz's in the $\mathrm{P}$ configuration.

\section{Friedel lattice (C1)}

The asymptotic form of the pairwise indirect electronic interaction, shown in Fig. 6, predicts a second minimum of only $8 \mathrm{meV}$, comparable to the calculated magnitude of surface-corrugation effects. However, in forming a lattice, there are multiple pairs contributing, effectively increasing the impact of this effect by a factor of 3 . Again, assuming a hexagonal lattice and using $A=0.25$, we get the experimental minimum of $a=10.2 \AA$ and a binding energy of 3 $\times 8 \mathrm{meV}=24 \mathrm{meV}$.

The overlayer-lattice parameter in phase $\mathrm{C} 1$ might be also influenced by the surface corrugation since the overlayer is essentially commensurate with a $4 \times 4$ copper unit cell, with a lattice parameter of $10.21 \AA$. The softness of the binding curves of Fig. 6 supports this idea. The diffusion barriers found in this study, however, suggests that it is hard to account for the stability of the $\mathrm{C} 1$ phase without including the surface-mediated interactions. Even if the scattering properties of the benzene adsorbate are closer to those of copper adsorbates $(A=0.08, \Theta=0.3 \pi)$, and therefore does not determine the overlayer-lattice parameter, the resulting barrier 
should contribute to the stability of this phase.

\section{CONCLUSIONS}

The low barrier to diffusion implies that benzene adsorbates can respond to weak interactions. The low barriers could, more generally, allow acene adsorbates to selforganize in response to either (or both) direct vdW interactions or (and) indirect surface-state-mediated interactions. There are several conditions for this to occur. The adsorption of the acene must be sufficiently weak that the process does not destroy the metallic surface state yet strong enough that the interaction it mediates can account for the regularity of the honeycomb network. For benzene molecules we have shown that these conditions are present and that the hexagonal-lattice ordering found in the $\mathrm{C} 1$ and $\mathrm{C} 2$ phases can be interpreted as arising from substrate-mediated and vdW binding, respectively.

\section{ACKNOWLEDGMENTS}

The authors thank SNIC (Swedish National Infrastructure for Computing) for access and for K.B.'s participation in the graduate school NGSSC. Work at University of Maryland was supported primarily by NSF under Grant No. CHE $07-$ 50334, with secondary support from NSF-MRSEC under Grant No. DMR 05-20471 and from the Computational Materials Science Network of DOE and ancillary support from the Center for Nanophysics and Advanced Materials (CNAM). Work at Chalmers was supported by the Swedish Research Council (Vetenskapsrådet VR) under Grant No. 621-2008-4346. The authors are grateful for exchanges with Daniel B. Dougherty. The authors thank Øyvind Borck for use of his code for Bader analysis. T.L.E. acknowledges extensive collaboration and helpful interactions with Ludwig Bartels and Kwangmoo Kim. *berland@chalmers.se

†einstein@umd.edu

*hyldgaar@chalmers.se

${ }^{1}$ Conjugated Polymer and Molecular Interfaces: Science and Technology for Photonic and Optoelectronic Applications, edited by W. R. Salaneck, K. Seki, A. Kahn, and J.-J. Pireaux (Marcel Dekker, New York, 2001).

${ }^{2}$ L. S. Hung and C. H. Chen, Mater Sci. Eng. R 39, 143 (2002).

${ }^{3}$ G. Pawin, K. L. Wong, K. Y. Kwon, and L. Bartels, Science 313, 961 (2006).

${ }^{4}$ S. D. Kevan and R. H. Gaylord, Phys. Rev. B 36, 5809 (1987).

${ }^{5}$ J. Repp, F. Moresco, G. Meyer, K.-H. Rieder, P. Hyldgaard, and M. Persson, Phys. Rev. Lett. 85, 2981 (2000); N. Knorr, H. Brune, M. Epple, A. Hirstein, M. A. Schneider, and K. Kern, Phys. Rev. B 65, 115420 (2002).

${ }^{6}$ K. H. Lau and W. Kohn, Surf. Sci. 75, 69 (1978); T. L. Einstein, ibid. 75, 161 (1978).

${ }^{7}$ T. L. Einstein, in Physical Structure of Solid Surfaces, edited by W. N. Unertl (Elsevier, Amsterdam, 1996), p. 577.

${ }^{8}$ P. Hyldgaard and M. Persson, J. Phys.: Condens. Matter 12, L13 (2000); P. Hyldgaard and T. L. Einstein, Europhys. Lett. 59, 265 (2002); P. Hyldgaard, T. L. Einstein, and M. Persson (unpublished).

${ }^{9}$ P. Hyldgaard and T. L. Einstein, Appl. Surf. Sci. 212-213, 856 (2003).

${ }^{10}$ V. S. Stepanyuk, A. N. Baranov, D. V. Tsivlin, W. Hergert, P. Bruno, N. Knorr, M. A. Schneider, and K. Kern, Phys. Rev. B 68, 205410 (2003).

${ }^{11}$ T. Munakata, T. Sakashita, and K. Shudo, J. Electron Spectrosc. Relat. Phenom. 88-91, 591 (1998).

${ }^{12}$ S. Lukas, S. Vollmer, G. Witte, and Ch. Wöll, J. Chem. Phys. 114, 10123 (2001)

${ }^{13}$ M. Xi, M. X. Yang, S. K. Jo, B. E. Bent, and P. J. Stevens, J. Chem. Phys. 101, 9122 (1994).

${ }^{14}$ D. Velic, A. Hotzel, M. Wolf, and G. Ertl, J. Chem. Phys. 109, 9155 (1998).

${ }^{15}$ D. B. Dougherty, P. Maksymovych, J. Lee, and J. T. Yates, Jr.,
Phys. Rev. Lett. 97, 236806 (2006)

${ }^{16}$ S. J. Stranick, M. M. Kamna, and P. S. Weiss, Science 266, 99 (1994).

${ }^{17}$ S. J. Stranick, M. M. Kamna, and P. S. Weiss, Surf. Sci. 338, 41 (1995).

${ }^{18}$ This small charge transfer was recognized already well before the implimentation of vdW-DW methods, e.g., by J. R. Lomas and G. Pacchioni, Surf. Sci. 365, 297 (1996).

${ }^{19}$ M. Dion, H. Rydberg, E. Schröder, D. C. Langreth, and B. I. Lundqvist, Phys. Rev. Lett. 92, 246401 (2004).

${ }^{20}$ T. Thonhauser, V. R. Cooper, S. Li, A. Puzder, P. Hyldgaard, and D. C. Langreth, Phys. Rev. B 76, 125112 (2007).

${ }^{21}$ D. C. Langreth, B. I. Lundqvist, S. D. Chakarova-Käck, V. R. Cooper, M. Dion, P. Hyldgaard, A. Kelkkanen, J. Kleis, Lingzhu Kong, Shen Li, P. G. Moses, E. Murray, A. Puzder, H. Rydberg, E. Schröder, and T. Thonhauser, J. Phys.: Cond. Mat. 21, 084203 (2009).

${ }^{22}$ P. Stoltze, J. Phys.: Condens. Matter 6, 9495 (1994).

${ }^{23}$ J. Repp, G. Meyer, K.-H. Rieder, and P. Hyldgaard, Phys. Rev. Lett. 91, 206102 (2003).

${ }^{24}$ M.-C. Marinica, C. Barreteau, M.-C. Desjonquères, and D. Spanjaard, Phys. Rev. B 70, 075415 (2004).

${ }^{25}$ H. Rydberg, N. Jacobson, P. Hyldgaard, S. I. Simak, B. I. Lundqvist, and D. C. Langreth, Surf. Sci. 532-535, 606 (2003).

${ }^{26}$ H. Rydberg, M. Dion, N. Jacobson, E. Schröder, P. Hyldgaard, S. I. Simak, D. C. Langreth, and B. I. Lundqvist, Phys. Rev. Lett. 91, 126402 (2003).

${ }^{27}$ A. Bilić, J. R. Reimers, N. S. Hush, R. C. Hoft, and M. J. Ford, J. Chem. Theory Comput. 2, 1093 (2006).

${ }^{28}$ D. C. Langreth, M. Dion, H. Rydberg, E. Schröder, P. Hyldgaard, and B. I. Lundqvist, Int. J. Quantum Chem. 101, 599 (2005).

${ }^{29}$ J. Harris, Phys. Rev. B 31, 1770 (1985).

${ }^{30}$ E. Ziambaras, J. Kleis, E. Schröder, and P. Hyldgaard, Phys. Rev. B 76, 155425 (2007).

${ }^{31}$ S. D. Chakarova-Käck, E. Schröder, B. I. Lundqvist, and D. C. Langreth, Phys. Rev. Lett. 96, 146107 (2006).

${ }^{32}$ K. Johnston, J. Kleis, B. I. Lundqvist, and R. M. Nieminen, 
Phys. Rev. B 77, 121404(R) (2008).

${ }^{33}$ S. D. Chakarova-Käck, Ø. Borck, E. Schröder, and B. I. Lundqvist, Phys. Rev. B 74, 155402 (2006).

${ }^{34}$ P. Sony, P. Puschnig, D. Nabok, and C. Ambrosch-Draxl, Phys. Rev. Lett. 99, 176401 (2007).

${ }^{35}$ N. Atodiresei, V. Caciuc, P. Lazić, and S. Blügel, Phys. Rev. Lett. 102, 136809 (2009).

${ }^{36}$ M. F. Crommie, C. P. Lutz, and D. M. Eigler, Science 262, 218 (1993).

${ }^{37}$ F. E. Olsson, M. Persson, A. G. Borisov, J.-P. Gauyacq, J. Lagoute, and S. Fölsch, Phys. Rev. Lett. 93, 206803 (2004).

${ }^{38}$ R. F. W. Bader, Atoms in Molecules: A Quantum Theory (Oxford University Press, Oxford, 1990).

${ }^{39}$ G. Henkelman, A. Arnaldsson, and H. Jónsson, Comput. Mater. Sci. 36, 354 (2006).

${ }^{40}$ J. P. Perdew, K. Burke, and M. Ernzerhof, Phys. Rev. Lett. 77, 3865 (1996).

${ }^{41}$ Y. Zhang and W. Yang, Phys. Rev. Lett. 80, 890 (1998).

${ }^{42}$ S. H. Vosko, L. Wilk, and M. Nusair, Can. J. Phys. 58, 1200 (1980).

${ }^{43}$ J. Kleis, E. Schröder, and P. Hyldgaard, Phys. Rev. B 77, 205422 (2008).

${ }^{44}$ J. Kleis, B. I. Lundqvist, D. C. Langreth, and E. Schröder Phys. Rev. B 76, 100201(R) (2007).

${ }^{45}$ K. H. Lau and W. Kohn, Surf. Sci. 65, 607 (1977).

${ }^{46}$ W. Kohn and K. H. Lau, Solid State Commun. 18, 553 (1976).

${ }^{47}$ J. K. Nørskov, in The Chemistry and Physics of Solid Surfaces, edited by D. A. King and D. P. Woodruff (Elsevier, Amsterdam, 1993), Vol. 6, Chap. 1, p. 1.

${ }^{48}$ M. A. Ruderman and C. Kittel, Phys. Rev. 96, 99 (1954); T.
Kasuya, Prog. Theor. Phys. 16, 45 (1956); K. Yosida, Phys. Rev. 106, 893 (1957).

${ }^{49}$ C. Kittel, in Solid State Physics, edited by F. Seitz, D. Turnbull, and H. Ehrenreich (Academic, New York, 1968), Vol. 22, p. 1; J. H. Van Vleck, Rev. Mod. Phys. 34, 681 (1962).

${ }^{50}$ V. B. Shenoy, Phys. Rev. B 71, 125431 (2005); E. H. Hwang and S. Das Sarma, Phys. Rev. Lett. 101, 156802 (2008).

${ }^{51}$ J. Friedel, Philos. Mag. 43, 153 (1952); G. D. Mahan, ManyParticle Physics, 2nd ed. (Plenum, New York, 1990), Sec. 4.1C.

${ }^{52}$ Open source code DACAPO, http://www.fysik.dtu.dk/CAMPOS/

${ }^{53} \mathrm{As}$ an interesting aside, we note that the vdW-DF differences in Bz-physisorption site preference shown in Fig. 4 originates almost entirely from difference in $E_{0}$ (from differences in kineticenergy repulsion) while the nonlocal-correlation energies differ by at most 2-3 meV between the sites FCC-I, HCP-I, BR-I, and TOP-I. Moreover, the fluctuations (numerical noise) in the evaluation of the nonlocal-correlation energy $E_{c}^{\mathrm{nl}}$ is very small, converged to the sub-meV level. Rather than being an issue with the $E_{c}^{\mathrm{nl}}$ evaluation, we have traced the origin of the small wiggles in the vdW-DF binding curve in the lower panel of Fig. 4 to $E_{0}$, especifically, the post-processing adjustment of the exchange from $\mathrm{PBE}$ to revPBE.

${ }^{54}$ L. W. Bruch, M. W. Cole, and E. Zaremba, Physical Adsorption: Forces and Phenomena (Oxford University Press, Oxford, 1997).

${ }^{55}$ E. D. Specht, M. Sutton, R. J. Birgeneau, D. E. Moncton, and P. M. Horn, Phys. Rev. B 30, 1589 (1984).

${ }^{56}$ D. A. Huse, Phys. Rev. B 29, 5031 (1984); S. Ostlund and A. N. Berker, ibid. 21, 5410 (1980). 